\title{
Purely radiative perfect fluids
}

\section{B Bastiaensen, H R Karimian, N Van den Bergh and L Wylleman}

Faculty of Applied Sciences TW16, Gent University, Galglaan 2, 9000 Gent, Belgium

\begin{abstract}
We study 'purely radiative' (i.e. $\operatorname{div} E=\operatorname{div} H=0$ ) and geodesic perfect fluids with non-constant pressure and show that the Bianchi class A perfect fluids can be uniquely characterized - modulo the class of purely electric and (pseudo-)spherically symmetric universes - as those models for which the magnetic and electric part of the Weyl tensor and the shear are simultaneously diagonalizable. For the case of constant pressure the same conclusion holds provided one also assumes that the fluid is irrotational.

PACS numbers: 04.20.Jb, 04.40.Nr
\end{abstract}

\section{Introduction}

In a previous paper [23] we discussed 'purely radiative' (i.e. $\operatorname{div} E=\operatorname{div} H=0$ ) irrotational dust spacetimes for which the magnetic part of the Weyl tensor is diagonal in the shear-electric eigenframe. We show that this analysis can be generalized to 'purely radiative' and geodesic perfect fluids. These are solutions of the Einstein field equations

$$
R_{a b}-\frac{1}{2} R g_{a b}=(\rho+p) u_{a} u_{b}+p g_{a b},
$$

where $u^{a}$ is the normalized 4-velocity, $\rho$ and $p$ the density and pressure of the fluid and $g_{a b}$ the spacetime metric, and for which we impose the additional restrictions (1) that the flow is geodesic: $\dot{u}_{a}=u_{a ; b} u^{b}=0$ and (2) that the electric and magnetic parts $E_{a b}$ and $H_{a b}$ of the Weyl tensor have vanishing spatial divergence. The spatial tensors $E_{a b}$ and $H_{a b}$ are hereby defined by

$$
E_{a b} \equiv C_{a c b d} u^{c} u^{d}=E_{<a b>} \quad H_{a b} \equiv \frac{1}{2} \varepsilon_{a c d} C^{c d}{ }_{b e} u^{e}=H_{<a b>},
$$

where $\varepsilon_{a b c}=\eta_{a b c d} u^{d}$ is the spatial projection of the spacetime permutation tensor $\eta_{a b c d}$ and $S_{<a b>}=h_{a}{ }^{c} h_{b}{ }^{d} S_{(c d)}-\frac{1}{3} S_{c d} h^{c d} h_{a b}$ is the projected, symmetric and trace-free part of $S_{a b}$, with $h_{a b}=g_{a b}+u_{a} u_{b}$ the spatial projector into the comoving rest space. The covariant spatial divergence and curl for tensors are defined by [13]:

$$
(\operatorname{div} S)_{a}=D^{b} S_{a b}, \quad \operatorname{curl} S_{a b}=\varepsilon_{c d(a} D^{c} S_{b)}{ }^{d},
$$

where the spatial part $D_{a}$ of the covariant derivative $\nabla_{a}$ is given by

$$
D_{a} A_{b \cdots}=h_{a}{ }^{c} h_{b}{ }^{d} \cdots \nabla_{c} A_{d \cdots}
$$


Gravitational radiation is covariantly described by the nonlocal fields $E_{a b}$ (the tidal part of the curvature), which generalizes the Newtonian tidal tensor, and by $H_{a b}$, which has no Newtonian analogue [6]. As such, $H_{a b}$ may be considered as the true gravitational wave tensor, since there is no gravitational radiation in Newtonian theory. However at least in the linear regime, as in electromagnetic theory, gravity waves are characterized by $H_{a b}$ and $E_{a b}$, where both are divergence-free but neither is curl-free [8]. For this reason spacetimes in which $\operatorname{div} E=0=\operatorname{div} H$ have been termed purely radiative [16].

Although, when considering the gravitational dynamics of matter in the full nonlinear regime, these conditions give rise to two independent chains of severe integrability conditions, it was remarked in [16] that both are satisfied by the Bianchi class A models. This motivated us to investigate purely radiative perfect fluids in their full generality.

As a first step we could show that in fact all the non-rotating dust models belong to Bianchi class A, provided we assume that they are not purely electric (in which case the term 'purely radiative' would be inappropriate anyway). In the purely electric case the exceptions were given by the Petrov type D spatially homogeneous and locally rotationally symmetric (LRS) metrics which are pseudo-spherically symmetric (and hence of Bianchi type $I I I$ ), or which are spherically symmetric and belong to the Kantowski-Sachs family of dust models and hence do not admit a 3 dimensional isometry group acting simply transitively on the hypersurfaces of homogeneity. For technical reasons we also assumed that $[\sigma, H]=[\sigma, E]=0$. Together with the fact that $[\sigma, E]=0$, which is a consequence of $\operatorname{div} H=0$, this implies that $\sigma_{a b}, E_{a b}$ and $H_{a b}$ can be simultaneously diagonalized (it is not necessary to assume that $[E, H]=0$, as a degenerate shear eigenplane also must be a degenerate $E$ eigenplane, see section 5 ).

When, on the contrary, one considers fluids with non-constant pressure (but still with geodesic flow), the spatial gradient of $p$ vanishes and the velocity becomes orthogonal to the $p=$ constant hypersurfaces, such that the flow is irrotational $[15,17]$. It is then a straightforward consequence of the assumptions $\operatorname{div} E=0$ and $[\sigma, H]=0$ that the spatial gradient of the matter density $\rho$ vanishes too. As stated in [15] it follows that $p, \sigma$ and $\theta$ are constants over the hypersurfaces of constant density and hence the resulting spacetimes satisfy the so called Postulate of Uniform Thermal Histories $[1,2,4,19]$. This seems to suggest that a local group of isometries exists, mapping the flow lines into each other (so called observational homogeneity). However not much progress has been made in this area and a detailed proof, or even a precise formulation of the conjecture that PUTH would lead to observational homogeneity, is still lacking. In fact it was suggested in [2] that any extra distinguishing mathematical property leading to observational homogeneity probably would have to be very complicated, involving at least third-order derivatives of the metric. An example of such an extra property is precisely the condition of being purely radiative, in the above sense of having $\operatorname{div} E=0=\operatorname{div} H$, which indeed enables us to show that the resulting spacetimes are spatially homogeneous and, more particularly, of Bianchi class A when we exclude the 'non-radiative' purely electric subcases of section 5.3. 


\section{Basic variables and equations}

We follow the notations and conventions of the Ellis-MacCallum orthonormal tetrad formalism [14]. Herein, the normalized 4-velocity $u^{a}$ plays the role of the timelike basis vectorfield of the tetrad $\left\{\mathbf{e}_{0}, \mathbf{e}_{\alpha}\right\}$. Greek indices take the values 1,2 and 3 and refer to tetrad components with respect to $\mathbf{e}_{\alpha}$.

The basic variables in the formalism are 24 independent linear combinations of the Ricci rotation coefficients or, equivalently, of the commutator coefficients $\gamma_{b d}^{a}$ associated with the tetrad; these are the objects $n_{\alpha \beta}$ and $a_{\alpha}$ defined by $\gamma_{\beta \delta}^{\alpha}=\epsilon_{\beta \delta \epsilon} n^{\epsilon \alpha}+\delta_{\delta}^{\alpha} a_{\beta}-\delta_{\beta}^{\alpha} a_{\delta}$, the components $\Omega_{\alpha}$ of the angular velocity of the triad $\mathbf{e}_{\alpha}$ with respect to the local 'inertial compass' and the components $\dot{u}_{\alpha}, \omega_{\alpha}$ and $\theta_{\alpha \beta}$ of the spatial kinematic quantities (acceleration vector, vorticity vector and expansion tensor respectively). We also use the decomposition $\theta_{\alpha \beta}=\sigma_{\alpha \beta}+1 / 3 \theta \delta_{\alpha \beta}$, with $\sigma_{a b}$ the trace-free shear tensor and $\theta$ the expansion scalar. It turns out that there is a slight advantage in using instead of the variables $n_{\alpha \beta}$ and $a_{\alpha}$ the connection coefficients $n_{\alpha}, q_{\alpha}$ and $r_{\alpha}$ related to the former by

$$
n_{\alpha+1 \alpha-1}=\left(r_{\alpha}+q_{\alpha}\right) / 2, \quad a_{\alpha}=\left(r_{\alpha}-q_{\alpha}\right) / 2, \quad n_{\alpha \alpha}=n_{\alpha+1}+n_{\alpha-1}
$$

(these expressions have to be read modulo 3 , so for example $\alpha=3$ gives $n_{12}=q_{3}+r_{3}$ ). For sake of simplicity we define $Z_{\alpha}, m_{\alpha}$ and $S_{\alpha}$ as the spatial gradients of $\theta, \rho$ and $\sigma^{2} \equiv \sigma^{a b} \sigma_{a b}$ respectively: $Z_{\alpha}=\partial_{\alpha} \theta, m_{\alpha}=\partial_{\alpha} \rho$ and $S_{\alpha}=\partial_{\alpha} \sigma^{2}$.

Together with the Einstein field equations, the basic equations are the Bianchi equations and the Ricci equations (part of which is equivalent with the Jacobi equations), which we have included in the appendix.

\section{Generalities}

From the Bianchi identity (36) (see appendix) and the assumption $\operatorname{div} H=0$ we get,

$$
(\operatorname{div} H)_{\alpha} \equiv D^{\beta} H_{\alpha \beta}=0 \Longleftrightarrow[E, \sigma]=0,
$$

which guarantees the existence of a frame wherein $E_{\alpha \beta}$ and $\sigma_{\alpha \beta}$ are both diagonal and which will be used henceforth.

Because all off-diagonal elements of $E_{\alpha \beta}$ vanish we derive from (32) that:

$$
\Omega_{1}\left(\sigma_{22}-\sigma_{33}\right)=\Omega_{2}\left(\sigma_{33}-\sigma_{11}\right)=\Omega_{3}\left(\sigma_{11}-\sigma_{22}\right)=0 .
$$

As a consequence the angular velocity $\Omega_{\alpha}$ can be assumed to be 0 : either all components automatically vanish, or, when e.g. the $\left(e_{2}, e_{3}\right)$ plane is a shear eigenplane, then $\Omega_{2}=\Omega_{3}=0$ and we can choose an extra rotation in this plane to make $\Omega_{1}=0$. The latter operation leaves the frame then fixed up to rotations in the $\left(e_{2}, e_{3}\right)$-plane for which the rotation angle $\varphi$ satisfies $\partial_{0} \varphi=0$. In fact, under a rotation about an angle $\varphi$ in the $\left(e_{2}, e_{3}\right)$-plane the quantities $\Omega$ transform as follows:

$$
\Omega_{1}^{\prime}, \Omega_{2}^{\prime}, \Omega_{3}^{\prime} \longrightarrow \Omega_{1}-\partial_{0} \varphi, \Omega_{2} \cos \varphi-\Omega_{3} \sin \varphi, \Omega_{2} \sin \varphi+\Omega_{3} \cos \varphi .
$$


The Raychaudhuri equation (43) and the conservation law (34) give us the evolution of respectively the expansion and the density:

$$
\begin{aligned}
& \partial_{0} \theta=-\frac{1}{3} \theta^{2}-\sigma^{2}-\frac{1}{2}(\rho+3 p) \\
& \partial_{0} \rho=-(\rho+p) \theta
\end{aligned}
$$

while (32) gives the evolution of the components $\sigma_{\alpha \alpha}$ :

$$
\begin{aligned}
& 3 \partial_{0} \sigma_{11}=\left(\sigma_{22}^{2}+\sigma_{33}^{2}-2 \sigma_{11}^{2}-2 \sigma_{11} \theta\right)-3 E_{11} \\
& 3 \partial_{0} \sigma_{22}=\left(\sigma_{33}^{2}+\sigma_{11}^{2}-2 \sigma_{22}^{2}-2 \sigma_{22} \theta\right)-3 E_{22}, \\
& 3 \partial_{0} \sigma_{33}=\left(\sigma_{11}^{2}+\sigma_{22}^{2}-2 \sigma_{33}^{2}-2 \sigma_{33} \theta\right)-3 E_{33} .
\end{aligned}
$$

The spatial derivatives of $\sigma_{\alpha \alpha}$ are obtained by the $(0 \alpha)$-Einstein field equations (44) and by the off-diagonal components of (29) :

$$
\begin{aligned}
& \partial_{1} \sigma_{11}=\frac{2}{3} Z_{1}+r_{1}\left(\sigma_{11}-\sigma_{22}\right)-q_{1}\left(\sigma_{11}-\sigma_{33}\right), \\
& \partial_{2} \sigma_{11}=-\frac{1}{3} Z_{2}+q_{2}\left(\sigma_{22}-\sigma_{11}\right)-H_{13}, \\
& \partial_{3} \sigma_{11}=-\frac{1}{3} Z_{3}+r_{3}\left(\sigma_{11}-\sigma_{33}\right)+H_{12},
\end{aligned}
$$

and similar expressions by cyclic permutation of the indices. Note that the $\operatorname{div} E=0$ condition relates the off-diagonal parts of $H_{\alpha \beta}$ to $m_{\alpha}$ by (35):

$$
\begin{aligned}
& m_{1}=3 H_{23}\left(\sigma_{33}-\sigma_{22}\right), \\
& m_{2}=3 H_{13}\left(\sigma_{11}-\sigma_{33}\right), \\
& m_{3}=3 H_{12}\left(\sigma_{22}-\sigma_{11}\right) .
\end{aligned}
$$

Using (5) we can now act with the commutator $\left[\partial_{0}, \partial_{\alpha}\right] \equiv-\left(\sigma_{\alpha \alpha}+\frac{1}{3} \theta\right) \partial_{\alpha}$ on $\rho$ to obtain the relation:

$$
\partial_{0} m_{\alpha}=-m_{\alpha}\left(\sigma_{\alpha \alpha}+\frac{4}{3} \theta\right)-(\rho+p) Z_{\alpha}, \quad \alpha=(1,2,3) .
$$

Propagating (8) along the fluid flow and substituting (6) and (9) gives the following expressions:

$$
\begin{aligned}
& 3\left(\sigma_{11}-\sigma_{22}\right) \partial_{0} H_{12}=\left(6 \sigma_{11}^{2}-6 \sigma_{22}^{2}-6 E_{22}-3 E_{33}+2 \sigma_{22} \theta-2 \sigma_{11} \theta\right) H_{12}+(\rho+p) Z_{3} \\
& 3\left(\sigma_{22}-\sigma_{33}\right) \partial_{0} H_{23}=\left(6 \sigma_{22}^{2}-6 \sigma_{33}^{2}-6 E_{33}-3 E_{11}+2 \sigma_{33} \theta-2 \sigma_{22} \theta\right) H_{23}+(\rho+p) Z_{1}(10) \\
& 3\left(\sigma_{33}-\sigma_{11}\right) \partial_{0} H_{13}=\left(6 \sigma_{33}^{2}-6 \sigma_{11}^{2}-6 E_{11}-3 E_{22}+2 \sigma_{11} \theta-2 \sigma_{33} \theta\right) H_{13}+(\rho+p) Z_{2}
\end{aligned}
$$

Provided we assume that also $H_{\alpha \beta}$ is diagonal in the $(\sigma, E)$-eigenframe, it follows directly from (8) and (9) or (10) that $m_{\alpha}=0$ and $Z_{\alpha}=0$ (regardless of the degeneracy of $\sigma_{\alpha \beta}$ ), giving a first hint that the corresponding spacetimes might be spatially homogeneous. To prove this we first find some information about the remaining part of the magnetic tensor.

Using the $\left[\partial_{0}, \partial_{\alpha}\right]$ commutators on $\theta$, we obtain from (31) and $Z_{\alpha}=m_{\alpha}=\partial_{\alpha} p=0$ that $S_{\alpha} \equiv \partial_{\alpha} \sigma_{a b} \sigma^{a b}=0$ and hence, using (7),

$$
\begin{aligned}
& r_{1}\left(\sigma_{11}-\sigma_{22}\right)^{2}-q_{1}\left(\sigma_{11}-\sigma_{33}\right)^{2}=0, \\
& r_{2}\left(\sigma_{22}-\sigma_{33}\right)^{2}-q_{2}\left(\sigma_{22}-\sigma_{11}\right)^{2}=0, \\
& r_{3}\left(\sigma_{11}-\sigma_{33}\right)^{2}-q_{3}\left(\sigma_{33}-\sigma_{22}\right)^{2}=0 .
\end{aligned}
$$


We also need the spatial derivatives of $E_{\alpha \alpha}$, which we can deduce from (35) and the off diagonal part of $(38)$ :

$$
\begin{aligned}
& \partial_{1} E_{11}=E_{11}\left(r_{1}-2 q_{1}\right)-E_{22}\left(r_{1}+q_{1}\right), \\
& \partial_{2} E_{11}=\left(E_{22}-E_{11}\right) q_{2}, \\
& \partial_{3} E_{11}=\left(E_{11}-E_{33}\right) r_{3} .
\end{aligned}
$$

We will treat the two cases of non-degenerate and degenerate shear now separately. Note that by (6) and (33) $\sigma_{\alpha \beta} \neq 0$, as otherwise the solutions would be FLRW.

\section{Non degenerate shear}

As the frame is invariantly defined, we first aim to proof that $r_{\alpha}$ and $q_{\alpha}$ are zero and that all spatial derivatives vanish. We first look at the evolution of $\sigma^{2}$ : from (31) it is easy to check that

$$
\partial_{0} \sigma^{2}=-\frac{4}{3} \theta \sigma^{2}-2 \sigma^{3}+2 E \cdot \sigma
$$

where $\sigma^{3} \equiv \sigma_{\gamma \alpha} \sigma_{\beta}^{\gamma} \sigma^{\alpha \beta}$ and $E \cdot \sigma \equiv E_{\alpha \beta} \sigma^{\alpha \beta}$.

Hence, acting with the commutator $\left[\partial_{0}, \partial_{\alpha}\right]$ on $\sigma^{2}$ we find:

$$
\partial_{0} S_{\alpha}=\frac{5}{3} \theta S_{\alpha}-\frac{4}{3} \sigma^{2} Z_{\alpha}-2 \partial_{\alpha} \sigma^{3}-2 \partial_{\alpha}(E \cdot \sigma)-S_{\alpha} \sigma_{\alpha \alpha}
$$

and hence, as $Z_{\alpha}=S_{\alpha}=0, \partial_{\alpha} \sigma^{3}+\partial_{\alpha}(E \cdot \sigma)=0$. Working out the latter componentwise and subsituting (12) and (7), results in three algebraic expressions. For $\alpha=1$ we get $r_{1}\left(\sigma_{22}-\sigma_{11}\right)\left[4 E_{22}+2 E_{33}-3 \sigma_{33}\left(\sigma_{22}-\sigma_{11}\right)\right]-q_{1}\left(\sigma_{33}-\sigma_{11}\right)\left[2 E_{22}+4 E_{33}-3 \sigma_{22}\left(\sigma_{33}-\sigma_{11}\right)\right]=0 .(15)$

Eliminating $r_{1}$ from (11) and (15), we find

$$
q_{1}\left(\sigma_{33}-\sigma_{11}\right)\left(\sigma_{22}-\sigma_{11}\right) \chi=0
$$

with

$\chi \equiv \frac{2}{3}\left[E_{11}\left(\sigma_{22}-\sigma_{33}\right)+E_{22}\left(\sigma_{33}-\sigma_{11}\right)+E_{33}\left(\sigma_{11}-\sigma_{22}\right)\right]-\left(\sigma_{11}-\sigma_{22}\right)\left(\sigma_{22}-\sigma_{33}\right)\left(\sigma_{33}-\sigma_{11}\right)$.

The whole calculation can be repeated for the cases $\alpha=2,3$ so that we finally have (as the shear is non-degenerate)

$$
q_{1} \chi=q_{2} \chi=q_{3} \chi=0 \text {. }
$$

If $q_{1}^{2}+q_{2}^{2}+q_{3}^{2} \neq 0$ it would follow from (17) that $\chi=0$, after which $\partial_{1} \chi=0$ leads to

$$
q_{1}\left(\sigma_{33}-\sigma_{11}\right)^{3}+r_{1}\left(\sigma_{22}-\sigma_{11}\right)^{3}=0,
$$

using (12) and (7). Eliminating $q_{1}$ from (18) and (11.a) gives

$$
-3 r_{1}\left(\sigma_{22}+\sigma_{33}\right)\left(\sigma_{33}-\sigma_{11}\right)^{2}\left(\sigma_{22}-\sigma_{11}\right)^{2}=0,
$$

which, as (11) implies that $r_{1}^{2}+r_{2}^{2}+r_{3}^{2} \neq 0$, leads to $\sigma_{11}=-\left(\sigma_{22}+\sigma_{33}\right)=0$. Similarly one would obtain $\sigma_{22}=\sigma_{33}=0$ and hence $\sigma_{\alpha \beta}=0$.

We conclude that for non-degenerate shear $q_{\alpha}=0$ and hence, by (11), also $r_{\alpha}=0$. This means that $a_{\alpha}=0$ and $n_{\alpha \beta}$ is diagonal. It is easy to check that all spatial derivatives of the rotation coefficients now vanish and hence [5] we obtain the non-degenerate spatially homogenous Bianchi class A models, i.e. types $I, I I, V I_{0}, V I I_{0}, V I I I$ and $I X$. 


\section{Degenerate shear}

From the degeneracy of the shear it is easily verified that $E_{\alpha \beta}$ must be degenerate too (32) and we may assume

$$
\begin{aligned}
\sigma_{\alpha \beta} & =\operatorname{diag}(-2 \sigma, \sigma, \sigma) \\
E_{\alpha \beta} & =\operatorname{diag}(-2 E, E, E) .
\end{aligned}
$$

For dust we can discard with the case of vanishing $E$ as a consequence of the non-existence of anti-Newtonian universes [24], but for non-constant pressure purely magnetic perfect fluids, which are necessarily of Petrov type I or D, do exist (we don't consider the conformally flat cases, as these uniquely reduce under the present assumptions to the FLRW models, see [9]): for type I the only possibility is the Bianchi $V I_{0}$ metric discussed in [22], while for type $\mathrm{D}$ the spacetime is locally rotationally symmetric class III [22]. As the fluid is non-rotating the solutions are then given precisely by the Bianchi type VIII or IX Lozanovski-Carminati metrics $[10,11,12]$, with a possible degeneracy to Bianchi type II (see below).

Henceforth we take $E$ non zero (both the type I and type D purely magnetic models above will reappear as $E=0$ special cases). Assuming that $H_{\alpha \beta}$ is diagonal in the $(\sigma, E)$-eigenframe, (11) implies

$$
r_{1}-q_{1}=r_{3}=q_{2}=0
$$

while the (23)-component of (30) shows that $r_{1}+q_{1}=0$ and hence $r_{1}=q_{1}=0$.

At this point the only variables having possibly non-vanishing spatial derivatives are $q_{3}, r_{2}$ and $n_{1}, n_{2}, n_{3}$. With the simplifications obtained so far, the (11)-Einstein equation (45), reduces to

$$
0=\frac{1}{3} \rho+\frac{1}{3} \theta\left(\sigma-\frac{1}{3} \theta\right)+\frac{2}{3} \sigma^{2}-E-n_{2} n_{3} .
$$

Eliminating now $\dot{E}_{\alpha \beta}$ from (37) for $\alpha=\beta=1$ and $\alpha=\beta=2$, we obtain

$$
0=H_{11}\left(-2 n_{1}+n_{2}-2 n_{3}\right)+H_{33}\left(-4 n_{1}-n_{2}-n_{3}\right),
$$

which in combination with (33) leads to

$$
\left(n_{1}+n_{2}+n_{3}\right)\left(n_{2}-n_{3}\right)=0 .
$$

We will discuss the three cases that follow from (21) separately; i.e. $n_{2} \neq n_{3}, n_{2}=n_{3} \neq 0$ and $n_{2}=n_{3}=0$. Notice that when $n_{2}=n_{3}$ the necessary and sufficient conditions for the fluid to be locally rotationally symmetric are automatically satisfied [7].

\section{1. $n_{2}-n_{3} \neq 0$}

By (21) we have $n_{1}+n_{2}+n_{3}=0$.

From (41) we get the evolution of $n_{\alpha}$, namely

$$
\begin{aligned}
& \partial_{0} n_{1}=\sigma\left(n_{2}+n_{3}\right)-\frac{1}{3} n_{1} \theta \\
& \partial_{0} n_{2}=-\sigma\left(n_{2}+3 n_{3}\right)-\frac{1}{3} n_{2} \theta \\
& \partial_{0} n_{3}=-\sigma\left(3 n_{2}+n_{3}\right)-\frac{1}{3} n_{3} \theta .
\end{aligned}
$$


Herewith we obtain, propagating $n_{1}+n_{2}+n_{3}$ along $\mathbf{e}_{0}$,

$$
0=\partial_{0}\left(n_{1}+n_{2}+n_{3}\right)=-3\left(n_{2}+n_{3}\right) \sigma
$$

and thus $n_{1}=0$ and $n_{2}=-n_{3} \neq 0$. Applying $\partial_{3}$ to (20) we find $\partial_{3} n_{2}=0$. On the other hand, calculating $\partial_{3} n_{2}$ from the (12)-Einstein equation (46) and the Jacobi equation (39), results in $\partial_{3} n_{2}=q_{3}\left(n_{3}-n_{2}\right)$. Hence we see that

$$
q_{3}\left(n_{3}-n_{2}\right)=0
$$

and thus $q_{3}=0$, as $n_{3} \neq n_{2}$ by assumption. It follows now that also $r_{2}=0$ as $\partial_{1} q_{3}=r_{2} n_{2}$ by (46) and (39). So $r_{\alpha}$ and $q_{\alpha}$ all become zero and we have

$$
\begin{aligned}
& n_{\alpha \beta}=\operatorname{diag}\left(0,-n_{2}, n_{2}\right) \quad\left(n_{2} \neq 0\right) \\
& a_{\alpha}=0 .
\end{aligned}
$$

This implies the vanishing of the spatial derivatives of all rotation coefficients $\left(\partial_{\alpha} \equiv 0\right)$ and we obtain a spatially homogeneous universe of Bianchi class A, type $V I_{0}$.

\section{2. $n_{2}=n_{3} \neq 0$}

From (20) and the (33)-Einstein equation (45) we can calculate

$$
\partial_{2} r_{2} \equiv \rho+3 \sigma^{2}-\frac{1}{3} \theta^{2}-n_{2}^{2}-2 n_{2} n_{1}+q_{3}^{2}+r_{2}^{2}+\partial_{3} q_{3}
$$

At this stage $n_{\alpha \beta}$ and $a_{\alpha}$ are given by

$$
n_{\alpha \beta}=\left[\begin{array}{ccc}
2 n_{2} & q_{3} / 2 & r_{2} / 2 \\
q_{3} / 2 & n_{1}+n_{2} & 0 \\
r_{2} / 2 & 0 & n_{1}+n_{2}
\end{array}\right], \quad a_{\alpha}=\left[\begin{array}{c}
0 \\
r_{2} / 2 \\
-q_{3} / 2
\end{array}\right] .
$$

Now $\partial_{1} n_{2}=0$ by the Jacobi equation (39), while $\partial_{2} n_{2}=0$ and $\partial_{3} n_{2}=0$ follow by combining respectively the (31)-Einstein equation (46) and the (12)-Einstein equation (46) with the Jacobi equation (39), so the spatial derivatives of $n_{2}$ vanish. We now try to obtain the Bianchi A condition $a_{\alpha}=0$ : remembering that we still have a rotational degree of freedom left (namely rotations in the (23)-plane about an angle $\varphi$ satisfying $\partial_{0} \varphi=0$ ), we use the following transformation formulas for the quantities $n_{\alpha \beta}$ and $a_{\alpha}$ : $n_{\alpha \beta} \rightarrow n_{\alpha \beta}^{r}, a_{\alpha} \rightarrow a_{\alpha}^{r}$ with

$$
\begin{aligned}
& n_{11}^{r}=n_{11} \\
& n_{22}^{r}=n_{33}^{r}=n_{22}-\partial_{1} \varphi \\
& n_{12}^{r}=\frac{1}{2}\left[\cos (\varphi)\left(\partial_{2} \varphi+q_{3}\right)-\sin (\varphi)\left(\partial_{3} \varphi+r_{2}\right)\right] \\
& n_{13}^{r}=\frac{1}{2}\left[\cos (\varphi)\left(\partial_{3} \varphi+r_{2}\right)+\sin (\varphi)\left(\partial_{2} \varphi+q_{3}\right)\right]
\end{aligned}
$$

and

$$
n_{23}^{r}=a_{1}^{r}=a_{2}^{r}-n_{13}^{r}=a_{3}^{r}+n_{12}^{r}=0 .
$$


A rotation making $a_{\alpha}=0$ and $n_{\alpha \beta}$ diagonal can then be obtained by $\partial_{2} \varphi=-q_{3}$ and $\partial_{3} \varphi=-r_{2}$, the integrability condition of which leads to $\partial_{1} \varphi=-\frac{K}{2 n_{2}}+n_{1}+n_{2}$, with

$$
K \equiv \rho+3 \sigma^{2}-\frac{1}{3} \theta^{2}+n_{2}^{2}
$$

One can check that the integrability conditions for the resulting system of pde's

$$
\begin{aligned}
& \partial_{0} \varphi=0 \\
& \partial_{1} \varphi=-\frac{K}{2 n_{2}}+n_{1}+n_{2} \\
& \partial_{2} \varphi=-q_{3} \\
& \partial_{3} \varphi=-r_{2}
\end{aligned}
$$

are identically satisfied. For the rotated variable $n_{1}$ we find $n_{1}+n_{2}=\frac{K}{2 n_{2}}$ : all spatial derivatives now vanish and we obtain the spatially homogeneous LRS Bianchi class A spacetimes of types $I I(K=0), V I I I(K<0)$ and $I X(K>0)$. An integrable subcase arises when $\rho=3 n_{2}^{2}-\theta(\sigma-\theta / 3)-6 \sigma^{2}$ : the electric part of the Weyl tensor is then 0 and we obtain the purely magnetic Lozanovski-Carminati metrics $[10,11,12]$, for which the Bianchi type is $V I I I$ or $I X$, reducing to type $I I$ for the $p=\rho / 5$ Collins-Stewart metric [3]. As $E_{\alpha \beta}=0$ the label 'purely radiative' is however not fully appropriate for these models.

Of course the above reasoning breaks down when $n_{1}=n_{2}=0$, a case which will be dealt with below.

\section{3. $n_{2}=n_{3}=0$}

Within the previous class the $n_{2}=n_{3}=0$ models are exceptional in the sense that they are 'purely electric' (by (33) one has $H_{\alpha \beta}=0$ ) and again cannot be termed 'purely radiative'. For completeness however we present the full details of the resulting special cases.

As in (5.2) one shows that a rotation exists under which $r_{2}, n_{1}$ and $\partial_{3} q_{3}$ become zero. By (20) this implies $q_{3}^{2}+K=0$, where $K$ is the same quantity as defined by (26). The rotation is now determined by a solution of the following system:

$$
\begin{aligned}
& \partial_{0} \varphi=0 \\
& \partial_{1} \varphi=n_{1} \\
& \partial_{2} \varphi=\cos (\varphi) \sqrt{-K}-q_{3} \\
& \partial_{3} \varphi=-\sin (\varphi) \sqrt{-K}-r_{2}
\end{aligned}
$$

for which again the integrability conditions are identically satisfied.

When $K<0$, one obtains

$$
n_{\alpha \beta}=\left[\begin{array}{ccc}
0 & \sqrt{-K} / 2 & 0 \\
\sqrt{-K} / 2 & 0 & 0 \\
0 & 0 & 0
\end{array}\right], \quad a_{\alpha}=\left[\begin{array}{c}
0 \\
0 \\
-\sqrt{-K} / 2
\end{array}\right]
$$


which is a LRS spatially homogenous Bianchi class B type III model [5].

When $K=0$ the rotation (28) results in $a_{\alpha}=0, n_{\alpha \beta}=0$, which gives a LRS spatially homogenous Bianchi class A type $I$ model (equivalent to Bianchi class A type $\left.V I I_{0}[5]\right)$.

A problem arises when $K>0: q_{3}, n_{1}$ and $n_{2}$ can still be made zero by a rotation, but it is no longer possible to choose a frame in which all spatial derivatives identically vanish. However the Cartan equations can now be integrated easily and one finds the Kantowski-Sachs universes with metric

$$
d s^{2}=-d t^{2}+Q(t)^{2} d r^{2}+P(t)^{2}\left[d \theta^{2}+\sin ^{2}(\theta) d \phi^{2}\right] .
$$

As is well known these do not admit a 3-dimensional isometry group acting simply transitively on the $t=$ constant hypersurfaces.

\section{Conclusion}

We have shown that the Bianchi class A perfect fluid models with non-constant pressure can be uniquely characterized as geodesic perfect fluid spacetimes which are purely radiative in the sense that the gravitational field satisfies $\operatorname{div} H=\operatorname{div} E=0$, under the assumption that also the magnetic part of the Weyl tensor $H_{\alpha \beta}$ is diagonal in the shearelectric eigenframe (i.e. $[H, \sigma]=[H, E]=0$ ). The only possible exception arises in the purely electric Petrov type D case of degenerate shear, where the allowed solutions are the Kantowski-Sachs universes or the pseudo-spherically symmetric Bianchi type III models $\left(K \equiv \rho+3 \sigma^{2}-\frac{1}{3} \theta^{2}>0\right.$ resp. $\left.K<0\right)$. The same conclusion holds for constant pressure provided one also assumes that the fluid is irrotational.

The next logical step is to investigate the case where $H, E$ and $\sigma$ are not simultaneously diagonalizable: it is hoped that here new classes of inhomogeneous and cosmologically interesting solutions might turn up, or, alternatively, that one would be able to demonstrate the remarkable result that the Bianchi class A spacetimes are the unique (modulo the LRS exceptions above) 'purely radiative' ones. Aside from the fact that such a characterization of the Bianchi A models would be quite neat, we feel that a further investigation of restrictions on $\operatorname{div} E$ and $\operatorname{div} H$ and of the role played by these third order quantities in general relativistic perfect fluids might shed a new light on the issues of PUTH and observational homogeneity.

\section{Appendix}

A first set of basic equations consists of Ricci and Bianchi equations [6], which we will use in a more formal sense and therefore give in their covariant form (assuming $\left.\dot{u}_{a}=\omega_{a}=0\right)$ :

\section{Ricci equations}

$$
\operatorname{curl} \sigma_{a b}-H_{a b}=0 \text {, }
$$


Purely radiative perfect fluids

$$
\begin{aligned}
& \dot{\sigma}_{<a b>}+\frac{2}{3} \theta \sigma_{a b}+\sigma_{c<a} \sigma_{b>}{ }^{c}+E_{a b}=0, \\
& \dot{\theta}+\frac{1}{3} \theta^{2}+\sigma_{a b} \sigma^{a b}+\frac{1}{2}(\rho+3 p)=0 .
\end{aligned}
$$

In their orthonormal tetrad form (with $E_{\alpha \beta}$ and $\sigma_{\alpha \beta}$ diagonal) the equations (29) and (30) read respectively

$$
\begin{aligned}
& E_{11}=-\partial_{0} \sigma_{11}+\frac{1}{3}\left(\sigma_{22}^{2}+\sigma_{33}^{2}-2 \sigma_{11}^{2}-2 \theta \sigma_{11}\right), \\
& \Omega_{1}\left(\sigma_{22}-\sigma_{33}\right)=0
\end{aligned}
$$

and

$$
\begin{aligned}
& H_{11}=\left(\sigma_{22}-\sigma_{11}\right) n_{3}+\left(\sigma_{33}-\sigma_{11}\right) n_{2}, \\
& H_{12}=\frac{1}{2} \partial_{3}\left(\sigma_{11}-\sigma_{22}\right)+\frac{1}{2}\left(\sigma_{33}-\sigma_{11}\right) r_{3}+\frac{1}{2}\left(\sigma_{33}-\sigma_{22}\right) q_{3}
\end{aligned}
$$

and similar equations obtained by cyclic permutation of the indices.

\section{Bianchi equations}

$$
\begin{aligned}
& \dot{\rho}=-(\rho+p) \theta, \\
& \operatorname{div} E_{a}=[\sigma, H]_{a}+\frac{1}{3} D_{a} \rho, \\
& \operatorname{div} H_{a}=-[\sigma, E]_{a}, \\
& \dot{E}_{<a b>}-\operatorname{curl} H_{a b}=-\theta E_{a b}+3 \sigma_{c<a} E_{b>}{ }^{c}-\frac{1}{2}(\rho+p) \sigma_{a b}, \\
& \dot{H}_{<a b>}-\operatorname{curl} E_{a b}=-\theta H_{a b}+3 \sigma_{c<a} H_{b>}{ }^{c},
\end{aligned}
$$

where the spatial dual of the commutator of tensors, $[S, T]_{a}$ is defined as $[S, T]_{a}=$ $\varepsilon_{a b c} S^{b d} T_{d}^{c}$.

A second set of equations consists of those Jacobi and Einstein equations which we use explicitly in our calculations and which we present in their orthonormal tetrad form (using the eigenframe of $\sigma_{a b}$ and $E_{a b}$ ), but now with the extra simplification $\dot{u}_{a}=\omega_{a}=0$ and $\Omega_{\alpha}=0$ (see section 3). Most of these equations appear in triplets which can be obtained from each other by cyclic permutation of the indices. For each equation we give only one representant.

Jacobi equations

$$
\begin{array}{ll}
(j 2-j 4): \quad & \partial_{1}\left(n_{2}+n_{3}\right)+\partial_{2} r_{3}+\partial_{3} q_{2}= \\
& \left(r_{1}-q_{1}\right)\left(n_{2}+n_{3}\right)+r_{3} r_{2}-q_{3} q_{2}, \\
(j 8-j 10): & \partial_{0}\left(r_{1}-q_{1}\right)-\partial_{1}\left(\sigma_{11}-\frac{2}{3} \theta\right)=-\left(r_{1}-q_{1}\right)\left(\sigma_{11}+\frac{1}{3} \theta\right), \\
(j 11-j 13): & \partial_{0}\left(n_{2}+n_{3}\right)=\left(2 \sigma_{11}-\frac{1}{3} \theta\right)\left(n_{2}+n_{3}\right), \\
(j 14-j 16): & \partial_{0}\left(r_{3}+q_{3}\right)+\partial_{3}\left(\sigma_{11}-\sigma_{22}\right)=-\left(\sigma_{33}+\frac{1}{3} \theta\right)\left(r_{3}+q_{3}\right) .
\end{array}
$$


Einstein equations

$$
\begin{aligned}
& (\operatorname{Ein} 00): \quad \partial_{0} \theta=-\left[\left(\sigma_{11}+\frac{1}{3} \theta\right)^{2}+\left(\sigma_{22}+\frac{1}{3} \theta\right)^{2}+\left(\sigma_{33}+\frac{1}{3} \theta\right)^{2}\right]-\frac{1}{2}(\rho+3 p), \\
& (\operatorname{Ein} 0 \alpha): \quad \frac{2}{3} \partial_{1} \theta-\partial_{1} \sigma_{11}=\frac{1}{2}\left(r_{1}+q_{1}\right)\left(\sigma_{22}-\sigma_{33}\right)-\frac{3}{2}\left(r_{1}-q_{1}\right) \sigma_{11}, \\
& (\operatorname{Ein} \alpha \alpha): \quad-\partial_{0}\left(\sigma_{11}+\frac{1}{3} \theta\right)-\partial_{1}\left(r_{1}-q_{1}\right)+\partial_{2} q_{2}-\partial_{3} r_{3}=\frac{1}{2}(p-\rho) \\
& +q_{2}\left(r_{2}-q_{2}\right)-r_{3}\left(r_{3}-q_{3}\right)-r_{1}^{2}-q_{1}^{2}+2 n_{2} n_{3}+\theta\left(\sigma_{11}+\frac{1}{3} \theta\right), \\
& (\operatorname{Ein} \alpha \beta): \quad-\partial_{1} r_{2}+\partial_{2} q_{1}-\partial_{3}\left(n_{1}-n_{2}\right)=q_{1}\left(r_{2}+q_{2}\right)+r_{2}\left(r_{1}+2 q_{1}\right) \\
& -\left(r_{3}-q_{3}\right)\left(n_{1}-n_{2}\right)+2 n_{3}\left(r_{3}+q_{3}\right) \text {. }
\end{aligned}
$$

Commutator relations

Finally we have the commutator relations, which can be written succinctly as

$$
\begin{aligned}
& {\left[\partial_{0}, \partial_{1}\right]=-\left(\sigma_{11}+\frac{1}{3} \theta\right) \partial_{1},} \\
& {\left[\partial_{1}, \partial_{2}\right]=q_{2} \partial_{1}+r_{1} \partial_{2}+\left(n_{1}+n_{2}\right) \partial_{3}}
\end{aligned} .
$$

\section{References}

[1] Bonnor W B, Ellis G F R 1986 Mon. Not. R. Astron. Soc 218, 605

[2] Bonnor W B, Pugh D J R 1987 S. Afr. J. Phys. 10, 169

[3] Collins C B, Stewart J M 1971 MNRAS 153, 419

[4] Collins C B 1990 Class. Quantum Grav. 7, 1983

[5] Ellis G F R, MacCallum M A H 1969 Commun. Math. Phys. 12, 108

[6] Ellis G F R 1971 Relativistic Cosmology, General Relativity and Cosmology ed R K Sachs (New York: Academic) p104-79

[7] Goode S W, Wainwright J 1986 GRG 18, 315

[8] Hogan P, Ellis G F R 1997 Class. Quantum Grav. 14, A171

[9] Kramer D, Stephani H, MacCallum MAH, Hoenselaers C, Herlt E 2003 Exact solutions of Einstein's field equations (Cambridge University Press)

[10] Lozanovski C 2002 Class. Quantum Grav. 19, 6377

[11] Lozanovski C, Carminati J 2003 Class. Quantum Grav. 20, 215

[12] Lozanovski C, Aarons M 1999 Class. Quantum Grav. 16, 4075

[13] Maartens R 1997 Phys. Rev. D 55, 463

[14] MacCallum M A H 1971 Cosmological Models from a Geometric Point of View (Cargèse) Vol 6 (New York: Gordon and Breach) p 61

[15] Raychaudhuri A K, Maity S R 1978 Phys. Rev. D 18, 10

[16] Sopuerta C F, Maartens R, Ellis G F R, Lesame W M 1999 Phys. Rev. D 60, 024006

[17] Synge J L 1937 Proc. London Math. Soc. 43, 376

[18] Trumper M 1965 Commun. Math. Phys. 6, 584

[19] Van den Bergh N 1989 Class. Quantum Grav. 6, 797

[20] van Elst H, Ellis G F R 1996 Class. Quantum Grav. 13, 1099

[21] Van den Bergh N, Wylleman L 2006 Class. Quantum Grav. 23, 3353

[22] Wylleman L, Van den Bergh N 2006 PRD 74, 084001

[23] Van den Bergh N, Bastiaensen B, Karimian R H, Wylleman L 2006 Purely radiative irrotational dust spacetimes XXIX Spanish Relativity Meeting, Universitat de les Illes Balears 
[24] Wylleman L 2006 Class. Quantum Grav. 23, 2727 